PROCEEDINGS OF THE

AMERICAN MATHEMATICAL SOCIETY

Volume 138, Number 9, September 2010, Pages 3171-3178

S 0002-9939(10)10410-9

Article electronically published on April 21, 2010

\title{
SECTIONAL LYAPUNOV EXPONENTS
}

\author{
ALEXANDER ARBIETO
}

(Communicated by Yingfei Yi)

\begin{abstract}
We define sectional Lyapunov exponents and use them to characterize sectional Anosov flows in terms of dominated splittings. In particular we improve a result of Sataev.
\end{abstract}

\section{INTRODUCTION}

The theory of hyperbolic systems appeared in the sixties, with the seminal works of Smale, introducing the horseshoe. The main feature of this dynamical system was the presence of complementary directions in the tangent bundle, one of them presenting a contractive behaviour and the other presenting an expansive behaviour. Since then the theory of hyperbolic dynamics grew, in particular to understand Anosov diffeomorphisms, which present this behaviour in the entire manifold, and expanding automorphisms, which present only expansion in all directions but are noninvertible.

Furthermore the theory evolved in directions beyond uniform hyperbolicity, and one such direction was the study of nonuniformly dynamical systems, with the aid of the so-called Lyapunov exponents. These exponents, when they exist and are nonzero, indicate asymptotic contraction or expansion along the orbit. The celebrated Oseledets theorem says that these exponents exist, and vary in a measurable way, in a set of full measure, for any invariant probability measure of the system.

It turns out that, for local diffeomorphisms, if all the Lyapunov exponents are positive in a set of total probability, i.e. a full measure set for any invariant measure, then the map is expanding, as shown in Alves-Araújo-Saussol [2] and Cao 7 . An analogous statement is true in the case of diffeomorphisms with an invariant dominated splitting: if all the Lyapunov exponents are negative in one subbundle and positive in the other in a set of total probability, then the diffeomorphism is Anosov.

In the case of vector fields, the hyperbolic theory also has a good understanding. In particular for Anosov flows, but now, the hyperbolic behaviour occurs transversally to the direction of the vector field. This automatically rules out the presence of singularities when the manifold is closed. Also, the Anosov flows share many

Received by the editors October 8, 2009

2010 Mathematics Subject Classification. Primary 37C10; Secondary 37D25.

Key words and phrases. Lyapunov exponents, sectional Anosov flows.

This work was partially supported by CNPq and by Faperj. The author thanks Prof. C. Morales for his useful comments.

(C)2010 American Mathematical Society Reverts to public domain 28 years from publication 
features of hyperbolic diffeomorphism, such as stability, spectral decomposition, etc.

In the presence of singularities there still exist flows which present some dynamical properties in a robust way and some weak form of hyperbolicity, where the so-called Lorenz attractor is the paradigmatic example [11, and which are called sectional Anosov flows [8. These flows occur in manifolds with boundary and also possess a dominated splitting, where one direction is uniformly contractive and the other uniformly expands the area of 2-planes inside it. It takes much effort to understand the dynamical properties of these flows; for instance, see [3, 4, 6] and 8.

In this paper, we characterize sectional Anosov flows in terms of the Lyapunov exponents in a set of total probability as in the above cited works, but now we also use sectional Lyapunov exponents to study these flows. These exponents are Lyapunov exponents of the cocycle defined by the second exterior power of the derivative; see the next section for precise definitions. The case of Anosov flows is included in our result, considering closed manifolds instead of only compact manifolds. Moreover we also extend a result found in Sataev's paper [10].

\section{Statement of the Results}

Let $M$ be a $C^{\infty} n$-dimensional connected compact Riemannian manifold; $M$ may have a boundary or not.

Let $\left\{\varphi_{t}: M \rightarrow M\right\}_{t \in \mathbb{R}}$ be a flow generated by a $C^{1}$-vector field $X$. We say that a probability measure $\mu$ is an invariant measure if $\mu\left(\varphi_{t}(A)\right)=\mu(A)$ for every measurable set $A$ and every $t \in \mathbb{R}$. We say that a subset $Y \subset M$ is a set of total probability if $\mu(Y)=1$ for every invariant measure $\mu$.

We will assume that $X$ is inwardly transverse to the boundary $\partial M$. The maximal invariant set of the flow is defined as $M(X)=\bigcap_{t \geq 0} \varphi_{t}(M)$. As usual, we say that a singularity is hyperbolic if the eigenvalues of the derivative of the vector field at the singularity have nonzero real part.

Definition 2.1. We say that the flow is sectional Anosov if every singularity is hyperbolic and there exists a continuous invariant splitting $T_{M(X)} M=E \oplus F$ over the maximal invariant set and constants $C>0$ and $\lambda>0$ such that for every $x \in M(X)$ and $t \geq 0$ :

(i) The splitting is not trivial: $E_{x} \neq\{0\}$ and $F_{x} \neq\{0\}$.

(ii) The splitting is dominated: $\left\|\left.D \varphi_{t}\right|_{E_{x}}\right\|\left\|\left.D \varphi_{-t}\right|_{F_{\varphi_{t}(x)}}\right\|<C e^{-\lambda t}$.

(iii) The subbundle $E$ is contracting: $\left\|D \varphi_{t}(x) v\right\| \leq C e^{-\lambda t}$, for every $v \in E_{x}-$ $\{0\}$.

(iv) The subbundle $F$ is sectionally expanding: For every 2-plane section $L \subset F$, if we denote $L_{x} \subset F_{x}$ to be the 2-plane in the subspace $F_{x}$, then

$$
\left|\operatorname{det}\left(D \varphi_{t}(x)\right)\right|_{L_{x}} \mid>C e^{\lambda t} .
$$

If only items (i) and (ii) are satisfied, we say that the splitting $E \oplus F$ is a dominated splitting over $M(X)$.

We observe that if the splitting $F$ decomposes as a continuous splitting $F=$ $\langle X\rangle \oplus G$, where $\langle X\rangle$ is the one-dimensional distribution generated by the vector field, then $\Lambda$ is a hyperbolic set. Indeed, since in the direction of the vector field the dynamics is an isometry, the expansion of the area must come from the $G$ subbundle, and this will imply that $G$ is uniformly expanding. In particular, in this 
case the continuity of the splitting implies that there are no singularities. Moreover, if $\partial M=\emptyset$, then $M(X)=M$ and we recover the definition of an Anosov flow. For more details, see, for instance, [5].

By Oseledet's theorem [9], for any invariant probability measure $\mu$ there exists a subset $Y$ with $\mu(Y)=1$ such that, for every $x \in Y$ there exists an invariant splitting:

$$
T_{x} M=\langle X\rangle \oplus E_{x}^{1} \oplus \cdots \oplus E_{x}^{s(x)},
$$

and the following limits exist:

$$
\lambda_{i}(x)=\lim _{t \rightarrow+\infty} \frac{1}{t} \log \left\|D \varphi_{t}(x) \cdot v\right\|, \text { for every } v \in E_{x}^{i}-\{0\}, i=1, \ldots, s(x) .
$$

Moreover, the functions $\lambda_{i}$ and $s$ are measurable and invariant by the flow; i.e. $s\left(\varphi_{t}(x)\right)=s(x)$ and $\lambda_{i}\left(\varphi_{t}(x)\right)=\lambda_{i}(x)$. Also, the splitting varies measurably. The numbers $\lambda_{i}(x)$ are called the Lyapunov exponents of the flow at the point $x$. If $E$ is a subbundle of the tangent bundle, then by "the Lyapunov exponents of $E$ " we mean the Lyapunov exponents of the nonzero vectors in $E$.

Let $V$ be a vector space. We denote by $\Lambda^{2} V$ the second exterior power of $V$, defined as follows. If $v_{1}, \ldots, v_{n}$ is a basis of $V$, then $\Lambda^{2} V$ is generated by $\left\{v_{i} \wedge v_{j}\right\}_{i \neq j}$. Any linear transformation $A: V \rightarrow W$ induces a transformation $\Lambda^{2} A: \Lambda^{2} V \rightarrow \Lambda^{2} W$. Moreover, $v_{i} \wedge v_{j}$ can be viewed as the 2-plane generated by $v_{i}$ and $v_{j}$ if $i \neq j$. See for instance [1] for more information.

Definition 2.2. The sectional Lyapunov exponents of $x$ along $F$ are the limits

$$
\lim _{t \rightarrow+\infty} \frac{1}{t} \log \left\|\Lambda^{2} D \varphi_{t}(x) . \widetilde{v}\right\|
$$

whenever they exist, where $\widetilde{v} \in \Lambda^{2} F_{x}-\{0\}$.

It turns out that if $\mu$ is an invariant probability measure, $Y$ is the subset given by Oseledet's theorem and $\left\{\lambda_{i}(x)\right\}_{i=1}^{s(x)}$ are the Lyapunov exponents, then the sectional Lyapunov exponents of a point $x \in Y$ are $\left\{\lambda_{i}+\lambda_{j}\right\}_{1 \leq i<j \leq s(x)}$. Moreover, condition (iv) in the definition of sectional Anosov flow can be obtained as follows. If $L_{x}$ is a 2-plane, then it can be seen as $\widetilde{v} \in \Lambda^{2}\left(F_{x}\right)-\{0\}$ of norm one. Hence, to obtain the sectional expansion, we only need to show that for some $\lambda>0$ and every $t>0$ the following inequality holds:

$$
\left\|\Lambda^{2} D \varphi_{t}(x) \cdot \widetilde{v}\right\|>C e^{\lambda t}
$$

Our main result is the following.

Theorem 2.3. Let $\left\{\varphi_{t}\right\}$ be a flow with a dominated splitting $T_{M(X)} M=E \oplus F$ over the maximal invariant set and such that every singularity is hyperbolic. The flow $\left\{\varphi_{t}\right\}$ is a sectional Anosov flow if and only if the Lyapunov exponents in the $E$ direction are negative and the sectional Lyapunov exponents in the $F$ direction are positive on a set of total probability. If the manifold has no boundary, the flow has no singularities and it is an Anosov flow.

We remark that this theorem improves a result found in [10]. First, let us recall the notation used by Sataev and set the scenario.

Assume that $M(X)$ has a dominated splitting $T_{M(X)} M=E \oplus F$, where the subbundle $E$ is uniformly contractive, and suppose that $\operatorname{dim} F=2$. Moreover, we assume that the fibers of $E$ are oriented. 
Letting $\Omega$ be the volume form of the manifold, we now define a 2 -form $\omega$ given by:

$$
\omega\left(w_{1}, w_{2}\right)=\Omega\left(w_{1}, \ldots, w_{n}\right) \text { for } w_{1}, w_{2} \in T_{x} M,
$$

where $w_{3}, \ldots, w_{n}$ form a parallelepiped of $E_{x}$ with unit volume and positive orientation. Hence the rank space of $\omega$ is the orthogonal complement of $E$.

Using the invariance of the subbundle $E$, Sataev proves that there exists a function $\theta(x)$ such that

$$
\dot{\omega}(x)=-\omega(x) D_{2}(x)+\theta(x) \omega(x),
$$

where $D_{2}$ acts as follows. Denote by $D(x)$ the matrix with elements $\left\{\frac{\partial X_{i}}{\partial x_{j}}\right\}$, where the $X_{i}$ are the coordinates of the vector field $X$ in the local coordinates $\left\{x_{j}\right\}$. Now, given two one-forms $p_{1}$ and $p_{2}$ and $p=p_{1} \wedge p_{2}$, we set

$$
p D_{2}=p_{1} D \wedge p_{2}+p_{1} \wedge p_{2} D
$$

Finally, $D_{2}$ is defined by linearity.

As noticed in [10, p. 54], it turns out that if $K_{x}$ is a small cone field containing the $F$-subbundle, then there exist constants $C_{1}$ and $C_{2}$, such that for any $v_{1}$ and $v_{2}$ in the cone, if we denote $A(L)$ as the area, defined by the Riemannian metric, of the plane generated by these two vectors, then we have

$$
C_{1}\left|\omega\left(v_{1}, v_{2}\right)\right| \leq A(L) \leq C_{2}\left|\omega\left(v_{1}, v_{2}\right)\right| .
$$

Letting $x \in M(X)$, the previous remark shows that the linear growth of the integral of the function $\theta$ along the orbit of $x$ is equivalent to the function $\log \left\|\Lambda^{2} D \varphi_{t}(x) \widetilde{v}\right\|$, where $\widetilde{v} \in \Lambda^{2} F_{x}$ has norm equal to one.

Indeed, Sataev proves that the following equality holds:

$$
\omega\left(\varphi_{t}(x)\right)=e^{\int_{0}^{t} \theta\left(\varphi_{s}(x)\right) d s} D_{* 2} \varphi_{t}(\omega(x)),
$$

where $D_{* 2}$ is the action of the differential on 2-forms. But the expansion of the area is equivalent to the existence of constants $C>0$ and $\gamma>0$ such that

$$
\omega\left(\varphi_{t}(x)\right)\left(D \varphi_{t}(x) v_{1}, D \varphi_{t}(x) v_{2}\right)>C e^{\gamma t} \omega\left(v_{1}, v_{2}\right) .
$$

Hence our theorem implies:

Corollary 2.4 (Lemma 2.12 of [10]). In this setting, the flow is sectional Anosov if and only if all singularities are hyperbolic and there exist constants $C \in \mathbb{R}$ and $D>0$ such that for every orbit $\varphi_{t}(x)$ and every $T>0$ the following inequality holds:

$$
\int_{0}^{T} \theta\left(\varphi_{t}(x)\right) d t>C+D T .
$$

Proof. The last statement obviously is a necessary condition for the flow to be sectional Anosov. Now, if the inequality holds for every orbit, then dividing by $T$ and taking limits. we have that the sectional Lyapunov exponents in the $F$ direction are positive for every orbit, in particular in a set of total probability; hence Theorem 2.3 applies.

We remark that our results also hold for sectional hyperbolic sets, i.e. with the same definition using a compact invariant set $\Lambda$ instead of the maximal invariant set $M(X)$. 


\section{Proof OF THE THEOREM}

In this section we prove Theorem 2.3, following the lines in [7].

Lemma 3.1. Let $f: M(X) \rightarrow \mathbb{R}$ be a continuous function such that $\int f d \mu<\lambda$ for any invariant probability measure $\mu$. Then for every $x \in M(X)$ there exists $t(x)>1$ such that

$$
\frac{1}{t(x)} \int_{0}^{t(x)} f\left(\varphi_{s}(x)\right) d s<\lambda .
$$

Proof. If not, there exists $x$ such that $\frac{1}{t} \int_{0}^{t} f\left(\varphi_{s}(x)\right) d s \geq \lambda$ for every $t>0$. Hence we define the measures $\mu_{t}=\frac{1}{t} \int_{0}^{t} \delta_{\varphi_{s}(x)} d s$, where $\delta_{x}$ is the Dirac measure at $x$. Now, we take $\mu=\lim _{k \rightarrow \infty} \mu_{t_{k}}$ as a cluster point of this sequence, with $t_{k} \rightarrow \infty$.

It is well known that $\mu$ is an invariant measure; moreover, since $f$ is continuous we have that

$$
\int f d \mu=\lim _{k \rightarrow \infty} \int f d \mu_{t_{k}}=\lim _{k \rightarrow \infty} \frac{1}{t_{k}} \int_{0}^{t_{k}} f\left(\varphi_{s}\right) d s \geq \lambda .
$$

Corollary 3.2. If $f: M(X) \rightarrow \mathbb{R}$ is a continuous function such that $\int f d \mu<\lambda$ for any invariant probability measure $\mu$, then there exists $T>0$ such that for any $t \geq T$ we have

$$
\frac{1}{t} \int_{0}^{t} f\left(\varphi_{s}(x)\right) d s<\lambda \text { for all } x \in M(X) .
$$

Proof. The previous lemma says that for every $x$ there exists some $t(x)>1$ and $\varepsilon(x)>0$ such that $\int_{0}^{t(x)} f\left(\varphi_{s}(x)\right) d s<t(x)(\lambda-\varepsilon(x))$.

Since $f$ is continuous, there exists some neighborhood $U_{x}$ such that for any $y \in U_{x}$ we have that $\int_{0}^{t(x)} f\left(\varphi_{s}(y)\right) d s<t(x)(\lambda-\varepsilon(x))$.

By compactness, we can cover $M$ by a finite number of such neighborhoods, say $U_{x_{1}}, \ldots, U_{x_{n}}$, and define $T_{0}=\max \left\{t\left(x_{1}\right), \ldots, t\left(x_{n}\right)\right\}$ and $\varepsilon=\min \left\{\varepsilon\left(x_{1}\right), \ldots, \varepsilon\left(x_{n}\right)\right\}$.

Now, we define by induction a sequence of functions $T_{k}: M \rightarrow\left\{1, \ldots, T_{0}\right\}$ for $k \geq 1$ as follows:

$$
\begin{aligned}
T_{0}(x) & =0, \\
T_{1}(x) & =\min \left\{t\left(x_{i}\right): x \in U_{x_{i}}, i=1, \ldots, n\right\}, \\
T_{k+1}(x) & =T_{k}(x)+T_{1}\left(\varphi_{T_{k}(x)}(x)\right) .
\end{aligned}
$$

Hence, for any $x \in M$ and $t>0$ there exists $k$ such that $T_{k}(x) \leq t \leq T_{k+1}(x)$, since $T_{k}(x) \rightarrow \infty$. In particular,

$$
\int_{0}^{t} f\left(\varphi_{s}(x)\right) d s \leq T_{k}(x)(\lambda-\varepsilon)+\|f\|_{0} T_{0} .
$$

Hence if $T=\frac{2\|f\|_{0} T_{0}}{\varepsilon}$ we have that, for any $x \in M$ and $t \geq T$,

$$
\frac{1}{t} \int_{0}^{t} f\left(\varphi_{s}(x)\right) d s<\lambda
$$

We say that a family of functions $\left\{f_{t}: M(X) \rightarrow \mathbb{R}\right\}_{t \in \mathbb{R}}$ is subadditive if for every $x \in M$ and $t, s \in \mathbb{R}$ we have that $f_{t+s}(x) \leq f_{s}(x)+f_{t}\left(\varphi_{s}(x)\right)$. The Subadditive Ergodic Theorem shows that the function $\bar{f}(x)=\liminf _{t \rightarrow+\infty} \frac{f_{t}(x)}{t}$ coincides with $\widetilde{f}(x)=$ $\lim _{t \rightarrow+\infty} \frac{1}{t} f_{t}(x)$ in a set of total probability. 
Remark 3.3. For any invariant measure $\mu$ we have that $\int \widetilde{f} d \mu=\lim _{t \rightarrow+\infty} \int \frac{f_{t}}{t} d \mu$.

Proposition 3.4. Let $\left\{t \mapsto f_{t}: M(X) \rightarrow \mathbb{R}\right\}_{t \in \mathbb{R}}$ be a continuous family of continuous functions which is subadditive and suppose that $\bar{f}(x)<0$ in a set of total probability. Then there exist constants $C>0$ and $\lambda<0$ such that for every $x \in M$ and every $t>0$ :

$$
e^{f_{t}(x)} \leq C^{-1} e^{\frac{\lambda t}{2}}
$$

Proof. The hypothesis says that $\tilde{f}(x)<0$ in a set of total probability. Hence, $\int \widetilde{f} d \mu<0$ for every invariant measure $\mu$. By the previous remark, for any invariant measure $\mu$ there exist $t(\mu) \in \mathbb{R}$ such that for every $t \geq t(\mu)$ :

$$
\int \frac{f_{t}}{t} d \mu<\frac{1}{2} \int \widetilde{f} d \mu
$$

Hence, there exists a neighborhood $U_{\mu}$ of $\mu$ in the weak-* topology such that if $\eta \in U_{\mu}$, then

$$
\int \frac{f_{t(\mu)}}{t(\mu)} d \eta<\frac{1}{4} \int \tilde{f} d \mu
$$

By weak compactness, the set of invariant measures can be covered by a finite number of such neighborhoods, say $U_{\mu_{1}}, \ldots, U_{\mu_{n}}$. Let $t(i)=t\left(\mu_{i}\right)$ for $i=1, \ldots, n$ (we can suppose $t(i)>1$ ) and define

$$
\lambda=\max _{i=1, \ldots, n}\left\{\frac{1}{4} \int \widetilde{f} d \mu_{i}\right\}<0 .
$$

In particular, for every invariant measure $\mu$ there exists some $i$ such that $\int f_{t(i)} d \mu<$ $\lambda t(i)$ and

$$
\begin{aligned}
\frac{1}{k t(i)} \int f_{k t(i)}(x) d \mu & \leq \frac{1}{k} \sum_{j=0}^{k-1} \frac{1}{t(i)} \int f_{t(i)}\left(\varphi_{j t(i)}(x)\right) d \mu \\
& =\frac{1}{k} \sum_{j=0}^{k-1} \frac{1}{t(i)} \int f_{t(i)}(x) d \mu \\
& <\lambda .
\end{aligned}
$$

Hence, if $L=t(1) t(2) \ldots t(n)$, then $\int f_{L} d \mu<\lambda L$.

Now, by the previous corollary applied to the continuous function $\frac{f_{L}}{L}$, there exists some $T_{0}$ such that, for any $t \geq T_{0}$ and any $x \in M$, we have

$$
\frac{1}{t} \int_{0}^{t} \frac{1}{L} f_{L}\left(\varphi_{s}(x)\right) d s<\lambda
$$

Since $f_{k L}(x) \leq \sum_{j=0}^{k-1} f_{L}\left(\varphi_{j L}(x)\right)$, then for every $0 \leq s<L$ we obtain

$$
f_{k L}(x) \leq f_{s}(x)+\left(\sum_{j=0}^{k-2} f_{L}\left(\varphi_{j L+s}(x)\right)\right)+f_{L-s}\left(\varphi_{(k-1) L+s}(x)\right) .
$$

Integrating, we get

$$
L f_{k L}(x) \leq \int_{0}^{L} \sum_{j=0}^{k-2} f_{L}\left(\varphi_{j L+s}(x)\right) d s+\left(\int_{0}^{L} f_{s}(x)+f_{L-s}\left(\varphi_{(k-1) L+s}(x)\right) d s\right) .
$$


Since $\left\{f_{t}\right\}$ is a continuous family of continuous functions over a compact manifold, we have that $B=\sup _{t \in[0, L]} \sup _{x \in M}\left|f_{t}(x)\right|<\infty$. Thus,

$$
f_{k L}(x) \leq \int_{0}^{(k-1) L} \frac{1}{L} f_{L}\left(\varphi_{s}(x)\right) d s+2 B .
$$

In particular,

$$
f_{k L}(x) \leq L(k-1) \lambda+2 B \text { if }(K-1) L>T_{0} .
$$

Now, take $t \geq T_{0}+2 L$ and write $t=k L+j$ where $0 \leq j<L$. So, $f_{t}(x) \leq$ $f_{k L}(x)+f_{j}\left(\varphi_{k L}(x)\right)$. Hence, $f_{t}(x) \leq L(k-1) \lambda+3 B$. Since $(k-1) L<t$, we have

$$
\frac{1}{t} f_{t}(x) \leq \lambda+\frac{3 B}{t}
$$

Taking $K=\max \left\{T_{0}+2 L, \frac{6 B}{-\lambda}\right\}$, we obtain that for every $x \in M$ and $t \geq K$ :

$$
\frac{1}{t} f_{t}(x) \leq \frac{\lambda}{2}
$$

Finally, define $C^{-1}=\sup _{s \in[0, K]}\left\{e^{f_{s}(x)}, 1\right\}$ and note that

$$
e^{f_{t}(x)} \leq C^{-1} e^{\frac{\lambda t}{2}} \text { for every } t>0 .
$$

Proof of Theorem 2.3. Let $\phi_{t}(x)=\log \left\|\left.D \varphi_{t}\right|_{E_{x}}\right\|$ and $\psi_{t}(x)=\log \left\|\left.\Lambda^{2} D \varphi_{-t}\right|_{F_{x}}\right\|$, both defined on $M(X)$ and continuous, since the subbundles are continuous.

Now, applying the proposition to the function $\phi_{t}$, we obtain that there exists some constant $C>0$ and $\lambda<0$ such that $e^{\phi_{t}(x)} \leq C^{-1} e^{\lambda t}$; hence $\left\|\left.D \varphi_{t}\right|_{E_{x}}\right\| \leq$ $C^{-1} e^{\lambda t}$ for every $x$, and this shows that $E$ is a contractive subbundle. Analogously, using $\psi_{t}$, we obtain constants $D>0$ and $\eta<0$ such that $\left\|\left.\Lambda^{2} D \varphi_{t}\right|_{F_{x}}\right\| \geq D e^{-\eta t}$, and this shows that $F$ is a sectionally expanding subbundle.

The proof is now complete.

\section{REFERENCES}

[1] Arnold, L., Random Dynamical Systems. Springer-Verlag, 2003. MR.1374107 (97a:58094)

[2] Alves, J., Araújo, V. and Saussol, B., On the uniform hyperbolicity of some nonuniformly hyperbolic systems. Proc. Amer. Math. Soc. 131, no. 4, 1302-1309, 2003. MR.1948124 (2003k:37046)

[3] Arbieto, A., Morales, C. and Senos, L., On the sensitivity of Sectional-Anosov Flows. Submitted to Communications on Mathematical Physics, 2009.

[4] Bautista, S., Morales, C., Existence of periodic orbits for singular-hyperbolic sets. Mosc. Math. J. 6, no. 2, 265-297, 406, 2006. MR2270614(2007m:37075)

[5] Bautista, S., Morales, C., Lectures on sectional Anosov flows. Submitted to Memoirs of the AMS, 2009.

[6] Bautista, S., Morales, C., Pacifico, M. J., On the intersection of homoclinic classes on singular-hyperbolic sets. Discrete Contin. Dyn. Syst. 19, no. 4, 761-775, 2007. MR2342271 (2008i:37056)

[7] Cao, Y., Non-zero Lyapunov exponents and uniform hyperbolicity. Nonlinearity 16, 14731479, 2003. MR 1986306 (2005g:37061)

[8] Morales, C., A., Sectional-Anosov systems. Monatsh. Math., to be published.

[9] Oseledets, V., A multiplicative ergodic theorem. Characteristic Ljapunov, exponents of dynamical systems. Trudy Moskov. Mat. Obšc. 19, 179-210, 1968. MR0240280(39:1629) 
[10] Sataev, E., Some properties of singular hyperbolic attractors, Math. Sbornik. 200:1, 27-80. 2009. MR2499676

[11] Viana, M., What's new on Lorenz strange attractors?, Math. Intelligencer, 22, no. 3, 6-19, 2000. MR:1773551 (2001h:37068)

Instituto de Matemática, Universidade Federal do Rio de Janeiro, P. O. Box 68530, 21945-970 RiO DE JANEIRO, BRAZIL

E-mail address: arbieto@im.ufrj.br 